
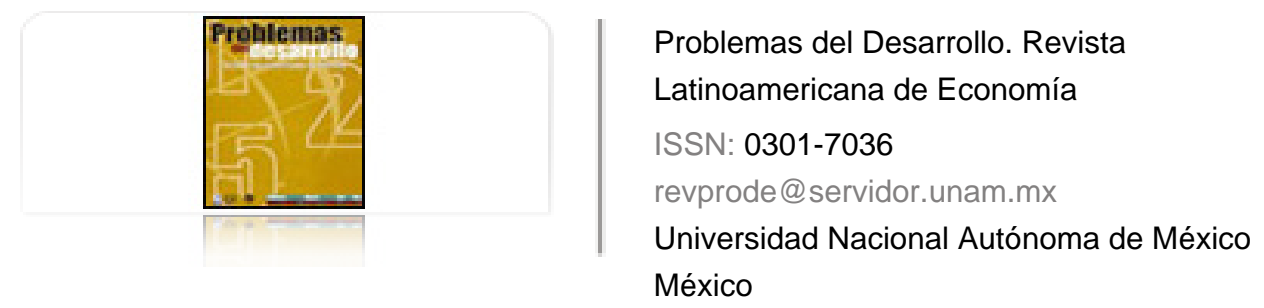

Encinas Ferrer, Carlos

NEOLIBERALISMO Y DISTRIBUCIÓN DEL INGRESO EN LOS ESTADOS UNIDOS DE AMÉRICA

Problemas del Desarrollo. Revista Latinoamericana de Economía, vol. 40, núm. 158, julio-septiembre, 2009, pp. 13-32

Universidad Nacional Autónoma de México

Distrito Federal, México

Disponible en: http://www.redalyc.org/articulo.oa?id=11820200001

- Cómo citar el artículo

- Número completo

- Más información del artículo

Página de la revista en redalyc.org

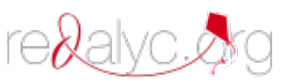

Sistema de Información Científica

Red de Revistas Científicas de América Latina, el Caribe, España y Portugal Proyecto académico sin fines de lucro, desarrollado bajo la iniciativa de acceso abierto 


\title{
NEOLIBERALISMO Y DISTRIBUCIÓN DEL INGRESO EN LOS ESTADOS UNIDOS DE AMÉRICA
}

\author{
Carlos Encinas Ferrer*
}

Fecha de recepción: 23 de febrero de 2009. Fecha de aceptación: 1 de agosto de 2009.

\section{Resumen}

La aplicación, a partir de 1980, de las políticas económicas neoliberales que buscaron fundamentalmente el desmantelamiento del Estado de bienestar, condujo al incremento en los niveles de concentración del ingreso y al aumento de la pobreza no sólo en los países subdesarrollados sino también en los industrializados. Este artículo presenta evidencia estadística de este fenómeno en los Estados Unidos de América, y en particular se ocupa de la manera en que el mismo afecta a los grupos más marginados de la sociedad capitalista moderna. Asimismo, se constata que en las décadas anteriores a 1980, época en la que se llevaron a cabo las políticas económicas y sociales del Estado de bienestar, se logró abatir los niveles de pobreza en los Estados Unidos y la concentración del ingreso permaneció estable; en contraste, luego de la aplicación de las políticas económicas neoliberales, se agudizó la concentración del ingreso en las capas altas de la sociedad.

Palabras clave: distribución del ingreso, pobreza, políticas neoliberales, grupos sociales marginados.

Clasificación JEL: I32, O15, P46.

* Investigador y académico de la Universidad De La Salle Bajío en León, México.

Correo electrónico: cencinas@delasalle.edu.mx 


\begin{abstract}
The application, from 1980, of neoliberal economic policies that sought fundamentally to dismantle the welfare State, led to an increase in the levels of income concentration and a rise in poverty, not only in the developing countries but also in the industrialized countries. The article presents statistical evidence of this phenomenon in the United States,

focusing especially on way in which this affects the most marginalized groups in a modern capitalist society. At the same time, it is clear that during the decades prior to 1980, an epoch in which welfare State economic and social policies were pursued, it was possible to reduce the levels of poverty in the United States, while income concentration was stable. In contrast, after the application of neoliberal economic policies, income concentration sharpened at the wealthiest end of society.

Keywords: income distribution, poverty, neoliberal policies, marginalized social groups.
\end{abstract}

\title{
Résumé
}

L'application, à partir de 1980, des politiques économiques néolibérales qui visèrent fondamentalement au démantèlement de l'État providence, a conduit à un niveau accru de concentration du revenu et à l'augmentation de la pauvreté non seulement dans les pays sous-développés mais aussi dans les pays industrialisés. Cet article présente la preuve statistique que ce phénomène se produit dans les EUA, et traite notamment de la manière dont il affecte les groupes les plus marginalisés de la société capitaliste moderne. Ainsi, il est constaté que dans les décennies antérieures aux années 80, époque où ont été menées les politiques économiques et sociales de l'État providence, les niveaux de pauvreté aux États-Unis ont pu être abaissés et la concentration du revenu restait stable; par contre, après la mise en application des politiques économiques néolibérales, on a vu s'y intensifier la concentration du revenu dans les couches sociales les plus élevées.

Mots clés: distribution du revenu, pauvreté, politiques néolibérales, groupes sociaux marginalisés.

\section{Resumo}

A aplicação, a partir de 1980, das políticas econômicas neoliberais que buscaram fundamentalmente o desmantelamento do estado de bem-estar, conduziu ao incremento nos níveis de concentração do ingresso e ao aumento da pobreza não só nos países subdesenvolvidos como também nos industrializados, como os EEUU. Este artigo apresenta evidência estatística deste fenômeno naquele país, e em particular se ocupa da maneira em que o mesmo afeta os grupos mais marginalizados da sociedade capitalista moderna. Constata-se também que nas décadas anteriores a 1980, época em que se levaram a cabo as políticas econômicas e sociais do estado de bem-estar, logrou-se um abatimento dos níveis de pobreza nos Estados Unidos e a concentração do ingresso permaneceu estável; em contraste, depois da aplicação das políticas econômicas neoliberais, agudizou-se a concentração do ingresso nas camadas altas da sociedade.

Palavras-chave: distribuição do ingresso, pobreza, políticas neoliberais, grupos sociais marginalizados. 


\section{Introducción}

a aplicación, a partir de 1980, de la política económica neoliberal, al mismo tiempo que desmantelaba gran parte del Estado de bienestar a través de una

serie de planes de ajuste y recortes presupuestarios en áreas como salud y seguridad social, redujo en forma importante los impuestos a los altos ingresos. Se daba como un hecho incuestionable "la teoría económica del derrame o del goteo de la riqueza de arriba abajo" (trickle down effect) ${ }^{1}$, según la cual el crecimiento económico generaría automáticamente mayor empleo, mayor ingreso y, por lo tanto, mayor consumo. Esa perspectiva omitió variables como la concentración del ingreso, el incremento en la productividad laboral que trajo la revolución tecnológica y el agotamiento que sufriría el mercado norteamericano como resultado lógico de la exportación de empleos industriales a países en los que era posible pagar menores sueldos y evitar costos ambientales. La teoría neoclásica, según la cual la remuneración del trabajo se da con base en su productividad marginal, reveló su inconsistencia en una mundialización en la que las únicas barreras que no se abren son las que impiden el libre movimiento de la mano de obra.

Tres décadas después, los resultados observados en la esfera mundial nos muestran una creciente concentración de la riqueza que en los países subdesarrollados se tradujo, ante el escaso crecimiento económico, en extensión de la pobreza, fenómeno que no fue privativo de las naciones menos desarrolladas y, por tener su origen en políticas que se aplicaron de manera global, también afectó a una economía altamente industrializada como la de los Estados Unidos de América (EEUU). Quedó clara la incapacidad del sistema económico imperante para generar una distribución más justa y equilibrada de la renta, aun al interior mismo de los centros de poder económico.

En este texto veremos cómo en los EEUU hubo un nulo crecimiento del ingreso real del quintil inferior de la población de más bajo ingreso, un escaso aumento en el siguiente quintil -estamos hablando de 120 millones de habitantes-y un aumento en el número absoluto de familias en niveles de pobreza.

1 Joseph Stiglitz, entrevista radiofónica realizada por Amy Goodman y Juan González, 5 de octubre de 2008. http://www.sinpermiso.info/textos/index.php?id=2095 


\section{El marco teórico}

Son múltiples los estudios en los que se ha tratado el tema de la concentración y la desigualdad del ingreso (income inequality) en la economía de los EEUU y la evolución de sus niveles de pobreza (Gabriel Kolko, 1962; Nan L. Maxwell, 1990; Folke Dovring, 1991; William H. H. Crown y Leonard F. Wheat, 1995; Paul Ryscavage, 1999). En los primeros años del presente siglo, el creciente interés por este tema se ha traducido en la aparición de nuevas investigaciones. En muchas de ellas la concentración del ingreso es considerada como un resultado lógico de las medidas neoliberales. Emmanuel Saez y Thomas Picketty han llevado a cabo el análisis de series largas que cubren un periodo que abarca desde 1917 hasta la fecha, y que nos presentan el comportamiento del ingreso por deciles poblacionales. Anthony Barnes Atkinson ha llevado a cabo estudios comparativos acerca de la desigual distribución del ingreso en diferentes países ${ }^{2}$. Especial importancia tiene el grupo de investigación del programa The Distribution of Income and Wealth en el Instituto Levy de Economía del Colegio Universitario Bard (The Levy Economics Institute of Bard College), en el que participan James K. Galbraith, Edward N. Wolff, Dimitri B. Papadimitriou, Ajit Zacharias, Selcuk Eren, Thomas Masterson, Barry Bluestone, Robert Haveman, Christopher Jencks, Susan E. Mayer, Branko Milanovic, Jacques Silber y Barbara Wolfe.

Una parte importante de estas investigaciones hace uso del Coeficiente de Gini y, por lo tanto, sus análisis se refieren a los niveles generales de inequidad en la distribución del ingreso y su evolución en el tiempo. Otros, analizan la distribución del ingreso de acuerdo con la división en quintiles o deciles de la población según sus niveles de ingreso.

En el presente estudio he escogido realizar el análisis a partir de la división en quintiles de las familias estadounidenses de acuerdo con su nivel de ingreso, pero desglosando cada una de estas divisiones según su origen étnico. El objetivo es tener un panorama más completo de la forma en que el origen social afecta a cada grupo, ya que es evidente que este factor introduce desigualdades hacia el interior de cada uno de los quintiles estudiados y, por lo tanto, no conviene analizarlos como una entidad homogénea en sí misma.

Por otra parte, es de especial interés conocer el comportamiento de las tendencias en cada nivel de ingreso y en cada segmento racial de los EEUU en dos épocas

2 La editorial Oxford University Press ha anunciado la puesta en venta en los primeros meses de 2010 del libro Top Incomes. A Global Perspective, en el que Atkinson y Picketty compilan una serie de escritos de diversos autores. 
perfectamente claras del desarrollo de la economía capitalista reciente: la anterior a 1980, previa a la aplicación de las políticas neoliberales por el gobierno de Ronald Reagan, y la que se da a partir de aquel año con el progresivo desmantelamiento de la economía de bienestar que acompañó a la apertura comercial y financiera, y con el dominio de las empresas transnacionales que marca la globalización.

En una entrevista reciente ${ }^{3}$, Joseph Stiglitz, ganador del Premio Nobel de Economía 2001, abunda en ejemplos de este fenómeno y coincide con una opinión que expresé en un artículo escrito en el año 2000, en el que mostré que la distribución del ingreso en los EEUU no había mejorado la situación económica del 40\% de la población de más bajo ingreso y, en cambio, sí había concentrado el ingreso en los estratos de mayor riqueza ${ }^{4}$. Lo anterior contradecía claramente las expectativas de una de las tesis del modelo económico neoliberal: la teoría económica del derrame o del goteo de la riqueza de arriba abajo ${ }^{5}$. Los datos que presento muestran claramente que esos postulados no se cumplieron en el quintil inferior del ingreso y que en el segundo quintil el crecimiento del ingreso fue inferior al del PIB en aquel país.

Actualizando y ampliando sustancialmente mis estudios iniciales y con el objetivo de conocer cuál fue el comportamiento de las tendencias prevalecientes antes y después de 1980, volví a navegar a través de internet hasta el servidor de la Oficina del Censo de Estados Unidos (US Census Bureau: http://www.census.gov). De esta magnífica central de información estadística he obtenido los datos que me han servido para corroborar aquellos argumentos y elaborar las gráficas que acompañan a este documento. Las bases de datos consultadas fueron las referentes al ingreso (income $)^{6}$ y a la pobreza (poverty) ${ }^{7}$.

\section{La pobreza en los Estados Unidos de América}

Para determinar quién es pobre en los Estados Unidos de América, la Oficina del Censo de aquel país utiliza un grupo de umbrales (thresholds) de ingreso monetario que varía de acuerdo con el tamaño de la familia y su composición. Estos límites o umbrales de pobreza son ajustados anualmente según el cambio porcentual en el promedio del Índice de Precios al Consumidor de aquel país.

3 Nathan Gardels, "Entrevista a Joseph Stiglitz”, Reporte índigo, núm. 23, México, Índigo Brainmedia, 2007, en http://www.reporteindigo.com/pdf/23/Reporteindigo.pdf

4 Carlos Encinas Ferrer, "Distribución del ingreso y pobreza en los EEUU", Revista contexturas, León, Guanajuato, Universidad Iberoamericana León, 2000, pp. 13-18.

5 Joseph Stiglitz, entrevista radiofónica, loc. cit.

6 US Census Bureau, http://www.census.gov/hhes/www/income/histinc/ineqtoc.html

7 US Census Bureau, http://www.census.gov/hhes/www/poverty/detailedpovtabs.html 
En la siguiente tabla se observan las características del ingreso correspondientes al segmento de la población considerado pobre por las estadísticas elaboradas en la Oficina del Censo de Norteamérica.

Tabla 1.

Umbral de pobreza en 2007 por tamaño de familia y número de hijos menores a 18 años (US dólares)

\begin{tabular}{|c|c|c|c|c|c|c|c|c|c|c|}
\hline \multirow[b]{2}{*}{$\begin{array}{l}\text { Tamaño de la } \\
\text { unidad familiar }\end{array}$} & \multirow[b]{2}{*}{$\begin{array}{l}\text { Umbrales } \\
\text { promedio } \\
\text { ponderados }\end{array}$} & \multicolumn{9}{|c|}{ Hijos menores de 18 años } \\
\hline & & Ninguno & Uno & Dos & Tres & Cuatro & Cinco & Seis & Siete & $\begin{array}{l}\text { Ochoo } \\
\text { más }\end{array}$ \\
\hline $\begin{array}{l}\text { Una persona (individuo } \\
\text { sin familia) }\end{array}$ & 10590 & & & & & & & & & \\
\hline Menos de 65 años & 10787 & 10787 & & & & & & & & \\
\hline 65 años o más & 9944 & 9944 & & & & & & & & \\
\hline Dos personas & 13540 & & & & & & & & & \\
\hline $\begin{array}{l}\text { Jefe de familia } \\
\text { menor a } 65 \text { años }\end{array}$ & 13954 & 13884 & 14291 & & & & & & & \\
\hline $\begin{array}{l}\text { Jefe de familia } \\
\text { mayor a } 65 \text { años }\end{array}$ & 12550 & 12533 & 14237 & & & & & & & \\
\hline Tres personas & 16530 & 16218 & 16689 & 16705 & & & & & & \\
\hline Cuatro personas & 21203 & 21386 & 21736 & 21027 & 21100 & & & & & \\
\hline Cinco personas & 25080 & 25791 & 26166 & 25364 & 24744 & 24366 & & & & \\
\hline Seis personas & 28323 & 29664 & 29782 & 29168 & 28579 & 27705 & 27187 & & & \\
\hline Siete personas & 32233 & 34132 & 34345 & 33610 & 33098 & 32144 & 31031 & 29810 & & \\
\hline Ocho personas & 35816 & 38174 & 38511 & 37818 & 37210 & 36348 & 35255 & 34116 & 33827 & \\
\hline Nueve personas o más & 42739 & 45921 & 46143 & 45529 & 45014 & 44168 & 43004 & 41952 & 41691 & 40085 \\
\hline
\end{tabular}

Fuente: traducción propia de la tabla del US Census Bureau. http://www.census.gov/hhes/www/poverty/threshld/thresh07.html

De acuerdo con estos criterios, el número de personas que vive en niveles de pobreza se ha estabilizado en alrededor de $12.5 \%$ del total, lo que en 2007 representaba 37661292 habitantes, cifra bastante mayor a la que generalmente se piensa que podría presentarse dentro de los umbrales de pobreza en una economía tan rica como la estadounidense.

En la gráfica 1 observamos que fue en la época de las políticas económicas keynesianas de bienestar social cuando los Estados Unidos pudo reducir sus niveles de pobreza desde más de $22 \%$ a sólo $11 \%$ de la población total. Sin embargo, a partir de la aplicación de las políticas económicas neoliberales, contrarias a la intervención del Estado a través de medidas de redistribución del ingreso, este porcentaje volvió 
a colocarse por arriba de las 15 unidades, y sólo gracias al prolongado periodo de crecimiento económico de la última década del siglo XX pudo recuperar, en el año 2000, los niveles de la década de 1970, pero sólo durante un breve lapso, para volver a situarse en niveles superiores a $12.5 \%$ de la población total en los años recientes. Será interesante observar el impacto que la actual crisis tendrá en los niveles de pobreza de nuestro país vecino.

Gráfica 1.

EEUU: \% de la población en umbrales de pobreza (1959-2007)

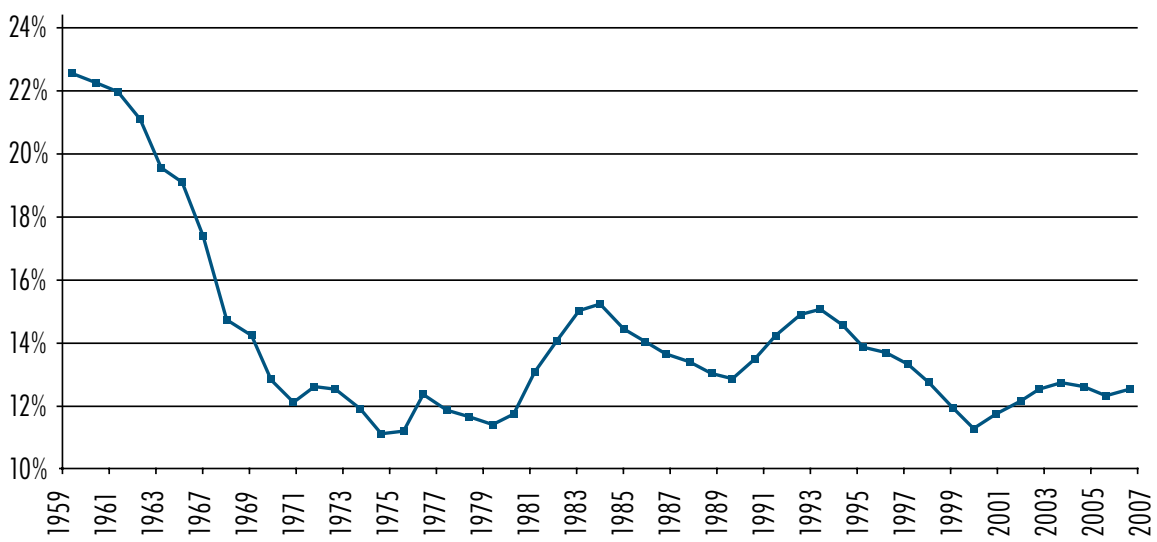

Fuente: elaboración propia con datos del US Census Bureau.

En la gráfica anterior observamos que el sistema económico norteamericano ha llegado a un "piso" de pobreza que ha sido incapaz de reducir. Ese $11 \%$ del total de la población en los estratos de más bajo ingreso parece haberse convertido en un límite infranqueable desde 1968.

La incapacidad del sistema para reducir la pobreza aparece en forma más dramática cuando se observan las cifras absolutas y no las porcentuales. En la gráfica 2 vemos que la cifra de 8 millones de familias en pobreza pudo reducirse a 5 millones en el periodo 1968-1979; sin embargo, con la aplicación de las políticas económicas de reducción del gasto público de carácter social, volvió a su origen.

En el año 2000 señalé ${ }^{8}$ que la disminución que entonces se observaba en la serie, que llegó a 11.3\% (6.48 millones de familias), con toda probabilidad volvería a estar

8 Carlos Encinas Ferrer, op. cit. 
por encima de su piso estructural ante la pronosticada recesión que ya en aquel momento amagaba con hacerse efectiva, ya que es obvia la relación entre desempleo y pobreza. En la gráfica 2 observamos que del año 2000 al 2004, el número de familias en umbrales de pobreza aumentó de poco menos de 6.5 millones de familias a un número cercano a 8 millones.

Gráfica 2.

EEUU: Millones de familias en niveles de pobreza (2007)

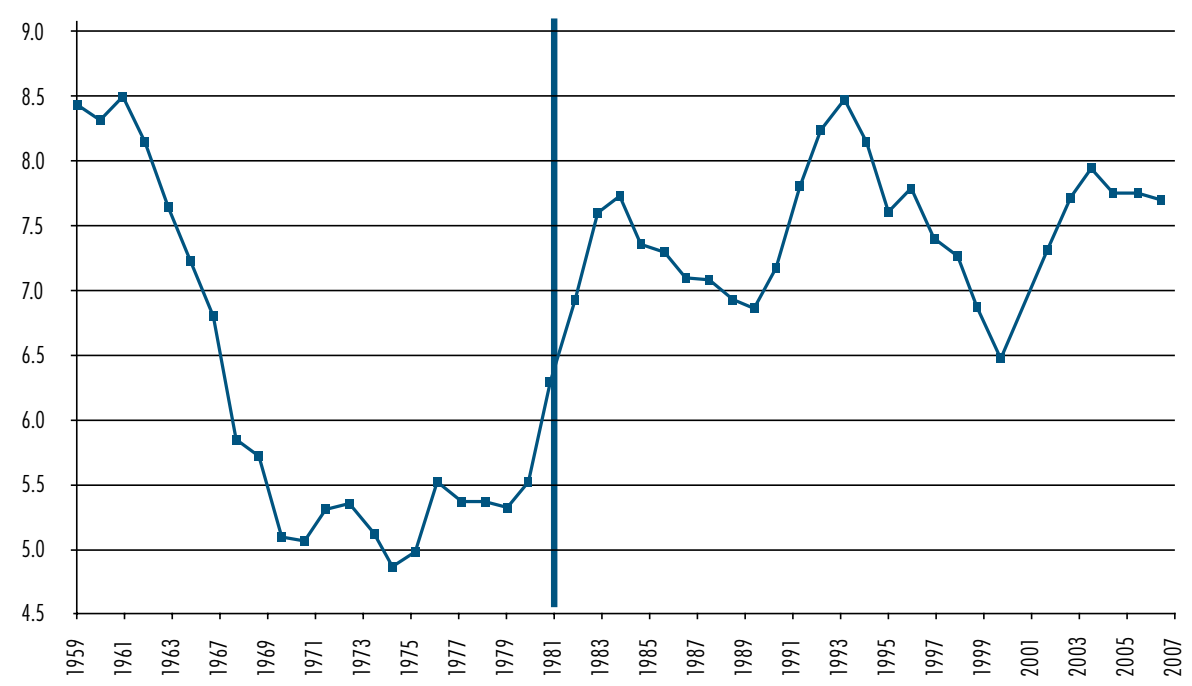

Fuente: elaboración propia con datos del US Bureau of the Census, Current Population Survey, Annual Social and Economic Supplements.

Un elemento relevante en el análisis de la pobreza en los EEUU es la composición étnica de su población. En la gráfica 3 observamos que mientras el $65.81 \%$ del total de la población norteamericana está formada por blancos no hispános, el porcentaje de este grupo disminuye a $43.01 \%$ en el segmento de pobreza. Por el contrario, el grupo hispano representa $15.38 \%$ de la población total pero su porcentaje asciende a $26.53 \%$ en el segmento de pobreza, mientras que los afroamericanos, el $12.61 \%$ de la población total, representan $24.78 \%$ de los pobres estadounidenses. Lo anterior refleja claramente la estructura social de la pobreza en los EEUU, categoría formada en más de un 50\% por hispanos y afroamericanos. 
Gráfica 3.

EEUU: Participación de tres grupos étnicos en la poblacion total y en el umbral de pobreza (2007)

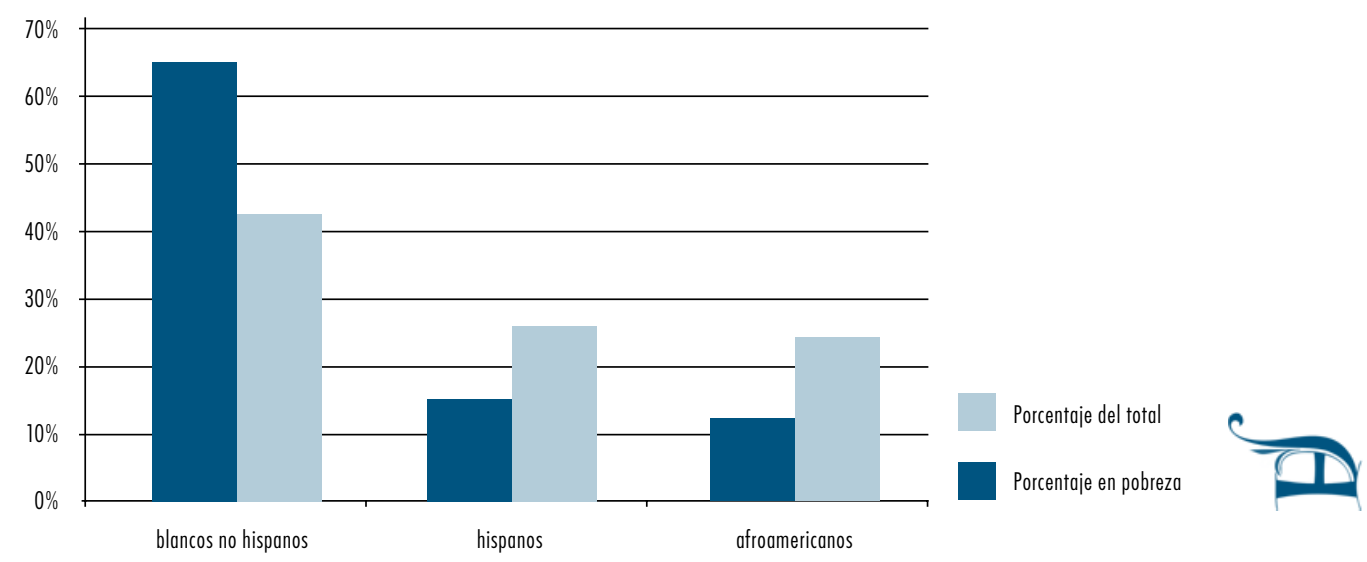

Fuente: elaboración propia con datos de Carmen DeNavas-Walt, Bernadette D. Proctor,

Jessica C. Smith, Income, Poverty, and Health Insurance Coverage in the United States: 2007,

US Census Bureau, Current Population Reports, P60-235, Washington, DC,

US Government Printing Office, 2008, p. 13.

\section{La distribución del ingreso en los Estados Unidos}

A continuación presento la forma en que se encuentra distribuido el ingreso entre los habitantes de la nación más poderosa del planeta, así como la manera en que dicha distribución evolucionó antes y después de 1980. Como señalé al inicio del presente trabajo, sigo la tradicional división del ingreso en quintiles, esto es, la división de la población total de los EEUU en cinco partes iguales, cada una de ellas compuesta de un $20 \%$ del total, lo que en 2007 representaba aproximadamente 60 millones de habitantes. El punto de partida es el $20 \%$ más pobre (quintil inferior) hasta el 20\% más rico (quintil superior). Las cifras se encuentran expresadas en dólares constantes de 2007.

En la gráfica 4 presento juntos los cinco quintiles que muestran la distribución del ingreso real de la población total de los Estados Unidos por familia. Las cifras van de 1966 a 2007, un total de 42 años, tiempo más que suficiente para que se manifiesten las tendencias de largo plazo. 
Gráfica 4.

EE.UU: Distribución media del ingreso familiar anual promedio (todas las razas). (dólares constantes de 2007)

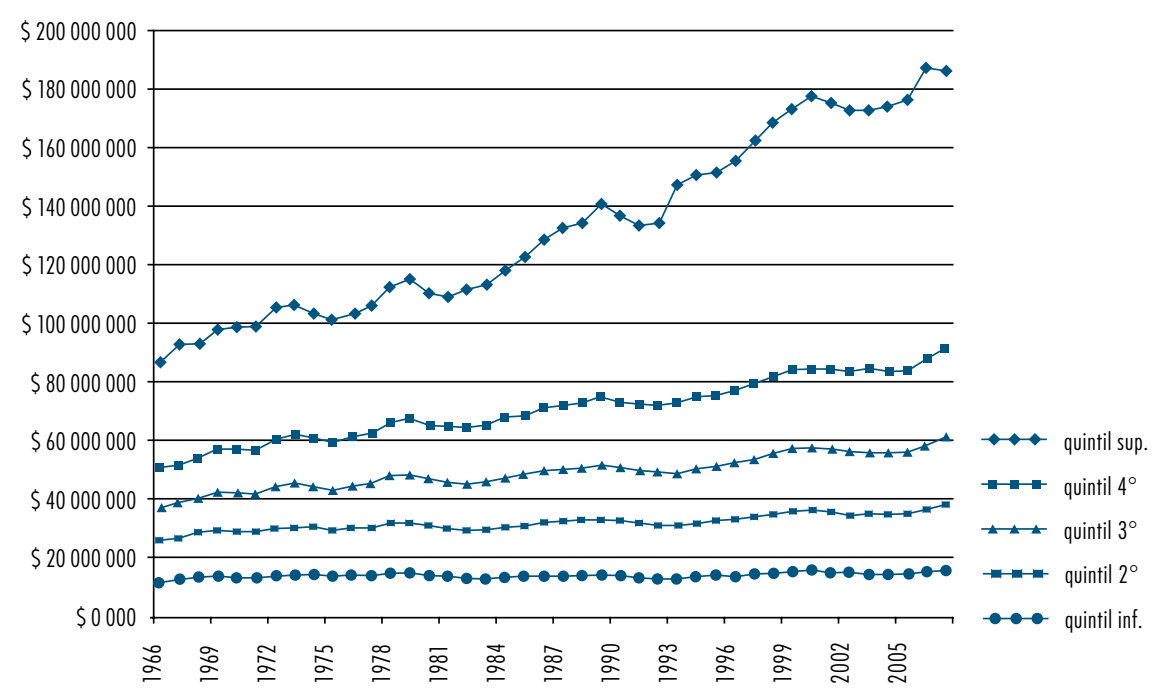

Fuente: elaboración propia con datos del US Census Bureau, Tablas F-3 All races. http://www.census.gov/hhes/www/income/histinc/ineqtoc.html

Destaca el escaso crecimiento en el ingreso real, medido a precios constantes de 2007, de los dos quintiles inferiores, los cuales representan $40 \%$ de la población total del país -aproximadamente 122 millones de habitantes-.

En el quintil inferior (véase gráfica 5), el ingreso aumentó $22.41 \%$ en 40 años, equivalente a $0.56 \%$ anual. El ingreso del segundo quintil, $31.5 \%$, un incremento anual de $0.79 \%$. Las cifras anteriores contrastan marcadamente con el incremento de $2.4 \%$ anual que permitió casi duplicar el ingreso del quintil superior en los 40 años estudiados. Es importante, sin embargo, desagregar la información y mostrar la distribución del ingreso según el origen étnico de la población, ya que una de las críticas más severas en contra de la globalización es que en mayor medida impacta negativamente a los grupos sociales que cuentan con menos capacidades laborales debido a la marginación social y económica.

Es interesante observar que $20 \%$ de la población blanca norteamericana de menores ingresos es la única en ese quintil que ha experimentado una mejoría, al pasar de un ingreso promedio per cápita de US \$16 878 (dólares constantes de 2007) en 1972, a US \$18 091 en 2007. Un incremento de 7.2\% en términos reales, equivalente a $0.21 \%$ de incremento promedio anual real en esos 35 años. Por otra parte, las 
Gráfica 5.

EEUU: Quintil inferior de la distribución del ingreso familiar anual promedio según origen étnico (dólares de 2007)

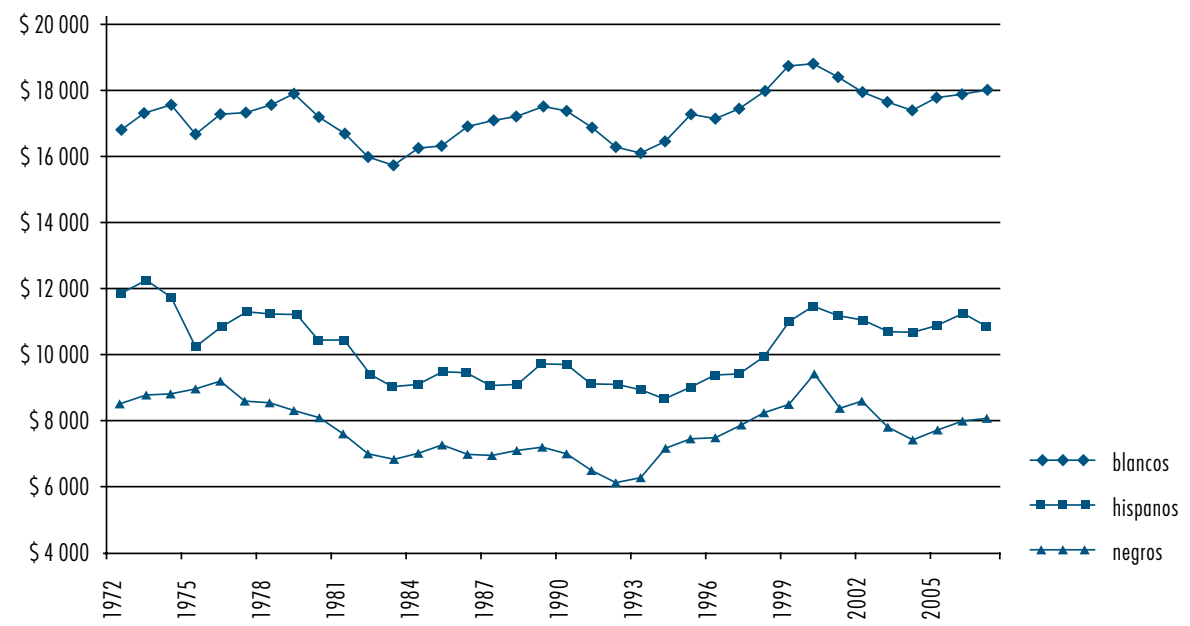

Fuente: elaboración con datos del US Census Bureau, Tablas F-3 por raza. http://www.census.gov/hhes/www/income/histinc/ineqtoc.html

familias de origen hispano pertenecientes al quintil inferior tenían, en 1972 -primer año en que la Oficina del Censo desagregó en sus estadísticas los datos de este grupo social étnico-, un ingreso medio equivalente a US \$11 870 (dólares de 2007), y en este último año el mismo se redujo a US \$10 902, un 8.2\% menor. En ese mismo periodo, las familias de origen afroamericano sufrieron una reducción de su ingreso medio en términos reales, que de US $\$ 8590$ pasó a $\$ 8079$, es decir, un descenso de 5.9 por ciento.

En la gráfica 6 observamos que la mayor proporción -más de 60\%- de la población en el quintil inferior de ingreso se encuentra por abajo del umbral de pobreza. Ya en la gráfica 5 vimos que la proporción de hispanos y negros en el umbral de pobreza es muy superior a la media nacional.

Por lo que se refiere al segundo quintil, que sumado al primero equivaldría hoy en día a alrededor de 122 millones de habitantes, la situación no ha mejorado mucho en los últimos 35 años. La gráfica 7 nos muestra que las familias de origen afroamericano se habían acercado al ingreso medio de las familias hispanas, pero en el periodo de la recesión económica posterior a 2000 volvieron a distanciarse. 
Gráfica 6.

EEUU: Porcentaje de individuos pobres en el quintil inferior

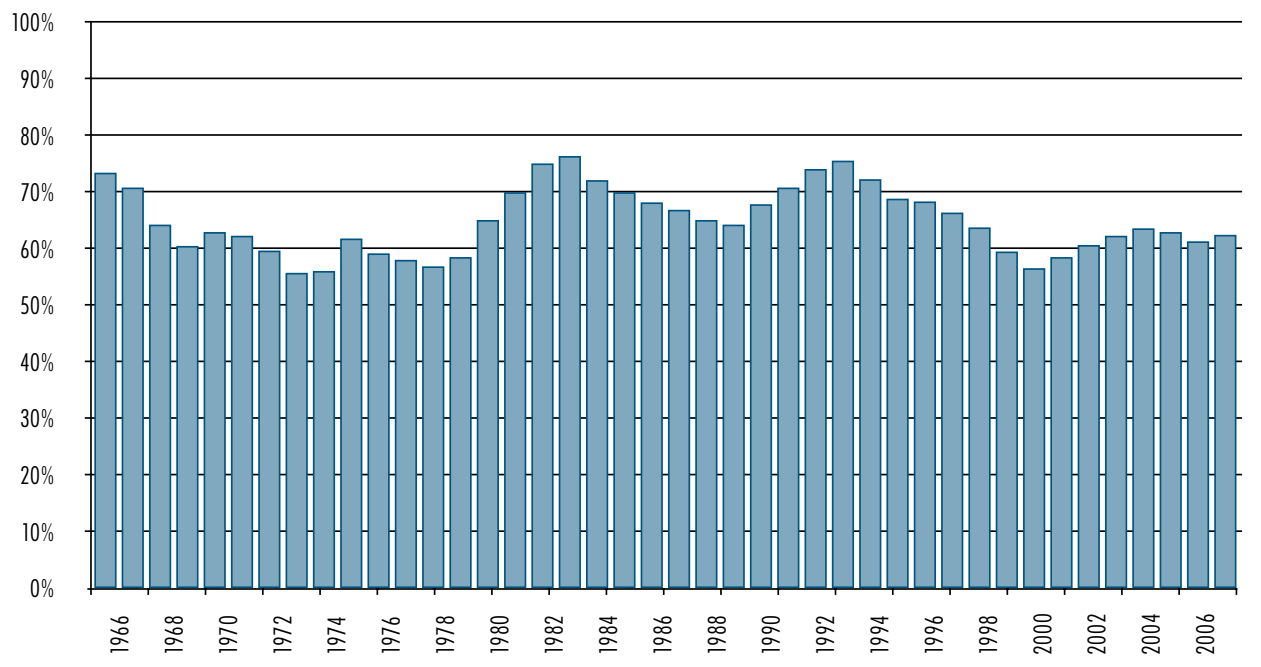

Fuente: elaboración propia con datos del US Census Bureau

Gráfica 7.

EEUU: Segundo quintil de la distribucion del ingreso familiar anual promedio segun origen étnico (dólares de 2007)

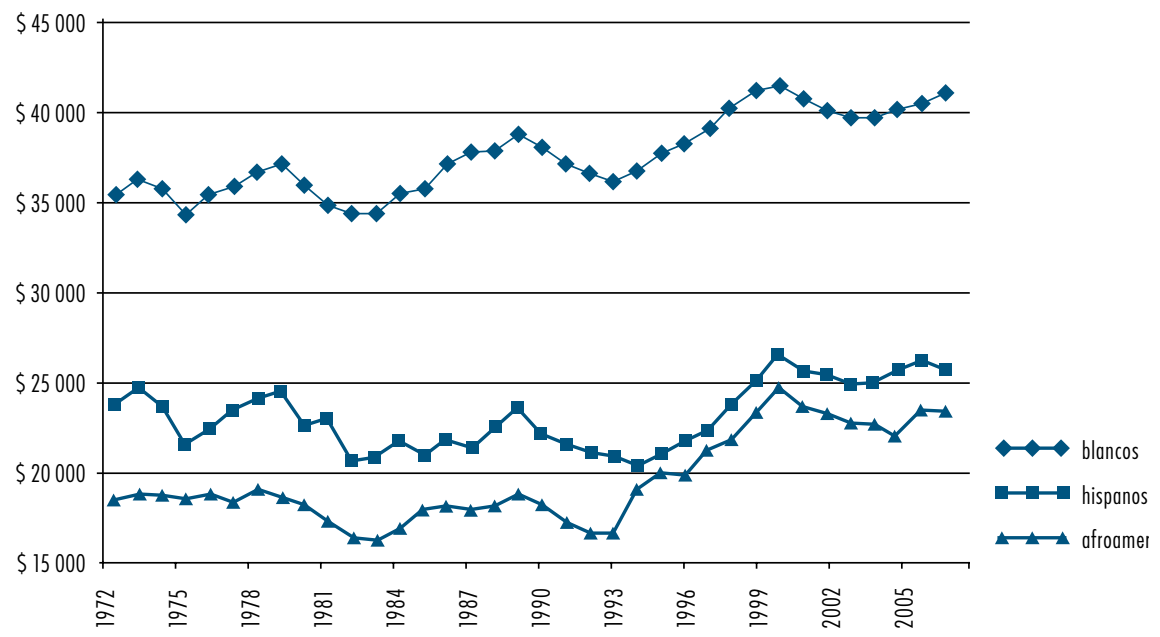

Fuente: elaboración con datos del US Census Bureau, Tablas F-3 por raza. http://www.census.gov/hhes/www/income/histinc/ineqtoc.html 
Al cabo de 35 años, el incremento del ingreso real fue, para las familias blancas de este quintil, de $15.32 \%$, al pasar de US \$35 543 anuales a US \$40 988 (una tasa anual de $0.44 \%$ ); para las de origen hispano, de 6.76\%, al pasar de US \$23 983 en 1972 a US \$25 604 en 2007 (un porcentaje anual real de 0.19\%); y las familias afroamericanas, cuyos ingresos fueron los que más aumentaron en este quintil, lograron un incremento de $25.37 \%$ (0.72\% anual), pasando de US \$18 580 a US \$23 294 dólares anuales. No obstante este crecimiento, este último grupo aún se encuentra US \$2 310 (dólares de 2007) por debajo del ingreso medio anual de las familias de origen hispano. Es importante señalar que nueve años antes, en 1998, esta diferencia era tan sólo de US \$1 805 (dólares reales de 2007).

Si tomamos en cuenta que entre 1972 y 2007 el PIB per cápita real (medido en dólares constantes de 2005$)^{9}$ creció a una tasa promedio de $2.74 \%$, queda claro que 122 millones de norteamericanos no se beneficiaron de ese crecimiento.

Todo lo anterior es congruente con el hecho de que "la globalización ha impuesto intensa presión en los salarios que reciben los trabajadores no calificados y la mano de obra menos especializada" ${ }^{10}$.

Esto contrasta con lo establecido por la teoría microeconómica, la cual presupone que personas con habilidades semejantes obtendrán igual salario, lo que se basa en la hipótesis de la teoría marginalista de que el salario es igual al producto marginal del trabajo o, lo que es lo mismo, los salarios los fijan los trabajadores menos productivos. Llama la atención que este supuesto se haya considerado una verdad incontrovertible, a pesar de la evidencia de que los salarios de las mujeres son mucho más bajos que los de los hombres, incluso en igualdad de condiciones, ya sean de productividad, origen racial o social.

Por otra parte, con la globalización parcial que hemos vivido ha aparecido otra deformación que desmiente al marginalismo y que es el resultado lógico de la existencia del libre flujo de capitales pero no el de la fuerza laboral, permitiendo establecer en los países subdesarrollados salarios menores a los que obtendrían los trabajadores de los países altamente industrializados pero con igualdad de eficiencia productiva. En otras palabras, la demanda global establece los precios de las mercancías mientras que el precio de la mano de obra se fija en mercados locales en los que no hay libre movilidad de la fuerza laboral.

9 US Department of Labor, Bureau of Labor Statistics, Office of Productivity and Technology, "Comparative real gross domestic product per capita and per employed person: 16 countries 1960-2007”, Washington, D.C., Bureau of Labor Statistics, 7 de julio de 2008. http://www.bls.gov/fls/home.htm

10 Nathan Gardels, loc. cit. 
Es en el quintil superior de la distribución del ingreso donde vemos hacia dónde ha ido a parar el crecimiento económico de los Estados Unidos de América. Todas las razas de este estrato incrementaron notablemente su ingreso medio anual, con lo que la concentración del ingreso se agudizó y mostró que tanto en los países más atrasados como en los más desarrollados se experimenta un proceso natural de distribución del ingreso, propio del modelo económico imperante.

Gráfica 8.

EEUU: Quintil superior de la distribución del ingreso familiar anual promedio según origen étnico (dólares de 2007)

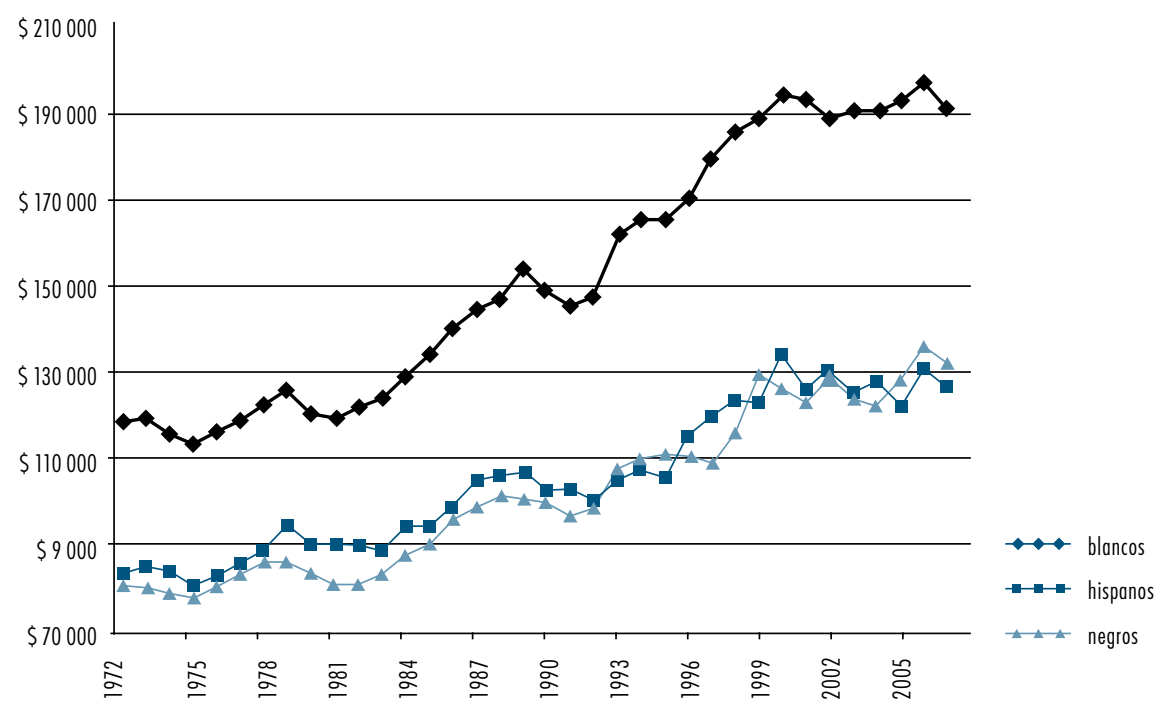

Fuente: elaboración propia con datos del US Census Bureau, Tablas F-3 por raza. http://www.census.gov/hhes/www/income/histinc/ineqtoc.html

El 20\% de la población blanca más rica vio crecer su ingreso $61.35 \%$ durante estos 35 años (una tasa anual de 1.75\%), al pasar de una media anual de US \$118 746 a US \$191 599. Las familias de origen hispano de este quintil superior incrementaron $50.73 \%$ sus ingresos medios anuales, pasando de US \$83 921 en 1972 a US \$126 496 en 2007 , un incremento promedio anual de $1.45 \%$. Las familias afroamericanas tuvieron un crecimiento en sus ingresos de 62.26\%, al pasar de US \$81 444 en 1972 a US \$132 147 en $2007,1.78 \%$ de incremento promedio anual, el mayor incremento porcentual en este segmento. 
En los años 2005, 2006 y 2007, las familias de origen hispano de este quintil superior perdieron su posición respecto de las familias afroamericanas. En el último año, el promedio de ingreso de aquéllas fue US \$5 651 inferior al de éstas.

Gráfica 9.

EEUU: 5\% superior de la distribución del ingreso familiar anual promedio según origen étnico (dólares de 2007)

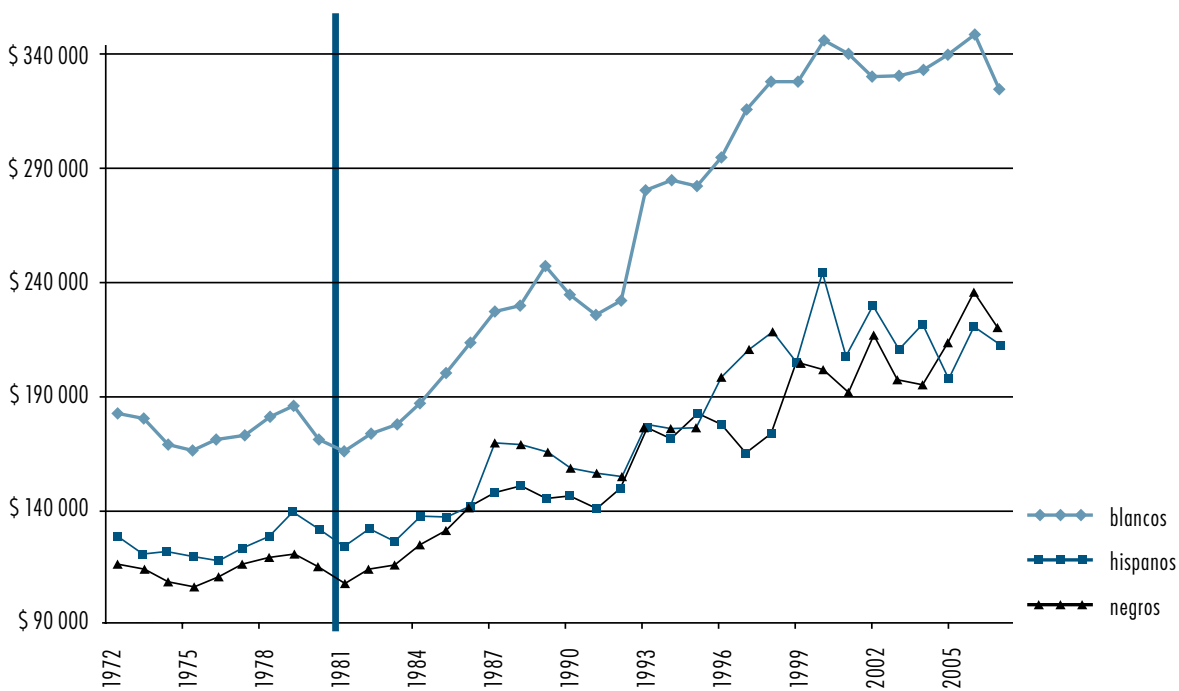

Fuente: elaboración con datos del US Census Bureau, Tablas F-3 por raza. http://www.census.gov/hhes/www/income/histinc/ineqtoc.html

En la gráfica 9 observamos que a partir de 1981, el ingreso se ha concentrado aún más en los niveles de renta elevada. Este punto de quiebre en la serie previa a 1981 aparece repetidas veces en mi investigación. En esta gráfica vemos que aunque la concentración del ingreso se dio en las tres razas, la pendiente es mayor en las familias blancas que en las de origen hispano y afroamericano. El ingreso del $5 \%$ de las familias con mayor riqueza (unos 15 millones de habitantes en 2007) ha aumentado en forma considerable, lo que permite constatar que han sido muy pocos los beneficiados por las medidas neoliberales. En el 5\% de la población blanca más rica, el ingreso medio anual pasó de US \$182 283 a US \$324 730 (en dólares de 2007), lo que representa $78.1 \%$ de incremento real (2.23\% de incremento anuales en términos reales). En el mismo segmento, las cifras de las familias hispanas muestran un incremento de US \$127 943 a US \$212 526 (en dólares constantes de 2007), esto es un ascenso de $66.1 \%$ (1.89\% anual). En el 5\% de las familias afroamericanas de 
mayores ingresos, la media anual creció $89.8 \%$ en esos 35 años, pasando de US $\$ 116388$ a US \$220 873 (en dólares constantes de 2007) es decir, 2.56\% anual. Resulta interesante observar que las familias hispanas de los dos últimos quintiles y, en especial, del 5\% de ingreso superior, en los últimos siete años fueron desplazadas de su posición por las familias afroamericanas. En 2007, estas últimas tuvieron un ingreso promedio superior de US $\$ 8347$ al de las familias de origen hispano. Igualmente, el ingreso promedio de las familias hispanas fue inferior al de las afroamericanas en los últimos tres años de la serie estudiada. Resulta evidente que en este nivel superior de ingreso, las familias hispanas resultaron más afectadas que los otros dos grupos sociales durante la recesión que siguió al año 2000.

La gráfica 10 es muy significativa sobre la manera en que ha evolucionado la concentración del ingreso en el 5\% de las familias de más alto ingreso de los EEUU. La tendencia lineal que se observa durante el lapso de 1966 a 1981, es sumamente clara y muestra que el porcentaje del ingreso total nacional que concentraba el 5\% más rico de la población disminuyó de $16 \%$ a $14.4 \%$. En contraste, a partir de la aplicación de la política neoliberal bajo el gobierno de Ronald Reagan, que condujo a reducir el Estado de bienestar, la tendencia lineal que acompañó al comportamiento de los datos en estos últimos 28 años nos muestra que aquel porcentaje se elevó y, en la actualidad, es superior a $20 \%$. Resalta el hecho de que las políticas neoliberales que coincidieron con la crisis de la deuda externa, no sólo golpearan a los países de menores recursos, sino también afectaran negativamente a las clases de menores ingresos en la nación norteamericana, beneficiando, en cambio, a las capas más ricas de esa nación.

La gráfica 11 nos muestra que la concentración elevada del ingreso en las familias más ricas de los EEUU no se modifica sustancialmente si en lugar de analizar al 5\% de mayor ingreso, lo hacemos con en el $20 \%$ superior (quintil número cinco). Entre 1966 y 1981, la proporción del ingreso en poder del $20 \%$ más rico de aquel país creció ligeramente al pasar de $40.53 \%$ a 41.06\%. Desde 1981, la tendencia de acumulación del ingreso en este quintil aumenta considerablemente, de tal manera que en 2007 se encontraba en $47.32 \%$, a pesar del descenso que aquel año presentó al bajar desde $48.5 \%$, derrumbe que lanzó señales admonitorias de la crisis que se acercaba.

Resulta impactante que a pesar de que los Estados Unidos posean una mejor estructura de reparto social de la riqueza, veamos que $20 \%$ de las familias de aquel país concentren en su poder casi $50 \%$ de todos los ingresos. 
Gráfica 10.

EEUU: Porcentaje del ingreso total en poder del $5 \%$ más rico de la población

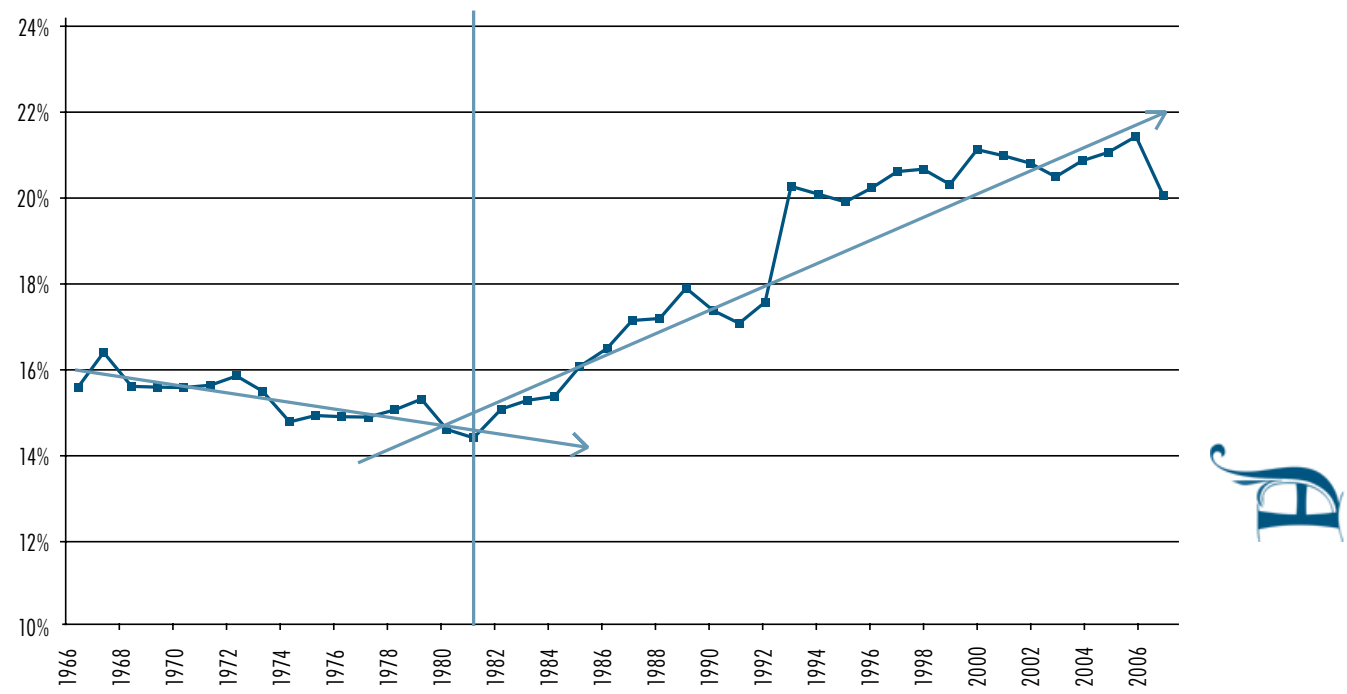

Fuente: elaboración propia con datos del US Census Bureau.

Ingresos promedio por familia y número de familias por quintil provienen respectivamente de http://www.census.gov/hhes/www/income/histinc/f03AR.html, y http://www.census.gov/hhes/www/ income/histinc/f01AR.html. Cálculos propios del ingreso total a partir de los datos de dichas tablas.

Gráfica 11.

EEUU: Porcentaje del ingreso total en poder del $20 \%$ más rico de la población

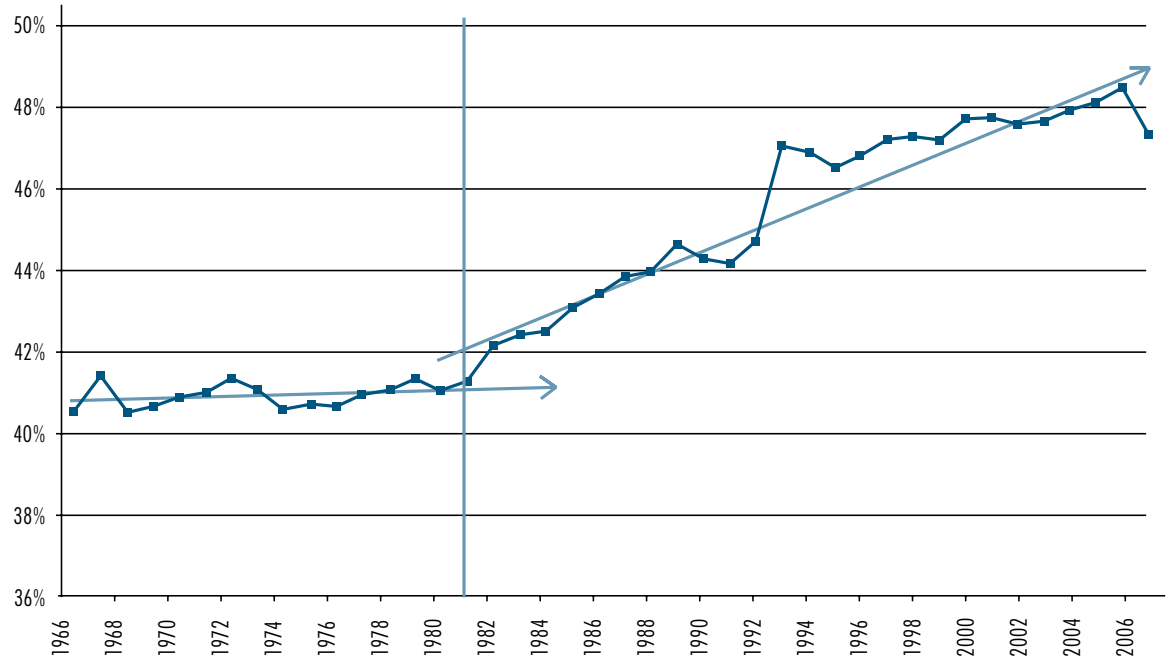

Fuente: elaboración propia con datos del US Census Bureau.

Ingresos promedio por familia y número de familias por quintil provienen respectivamente de http://www.census.gov/hhes/www/income/histinc/f03AR.html, y http://www.census.gov/hhes/www/ income/histinc/f01AR.html. Cálculos propios del ingreso total a partir de los datos de dichas tablas. 
En la gráfica 12 observamos la evolución del monto total del ingreso del 5\% de las familias más ricas de los EEUU comparado con la del $40 \%$ de las familias de menor ingreso. Destaca, una vez más, 1981 como parte aguas de la concentración del ingreso en los Estados Unidos.

Es obvio que esta concentración del ingreso en un porcentaje menor de la población tiene un impacto sobre el mercado y su capacidad para asignar eficientemente los recursos. Cuando 15 millones de personas (5\% de la población de los EEUU en 2007) tienen un poder de compra $45.6 \%$ mayor que 122 millones (40\% de la población), es necesario recurrir al crédito para poder vaciar los mercados y suplir el abismo existente entre una capacidad productiva ampliada enormemente por el desarrollo tecnológico y una estructura de distribución del ingreso que no le corresponde.

Gráfica 12.

EEUU: Ingreso total en millones de dólares constantes de 2007

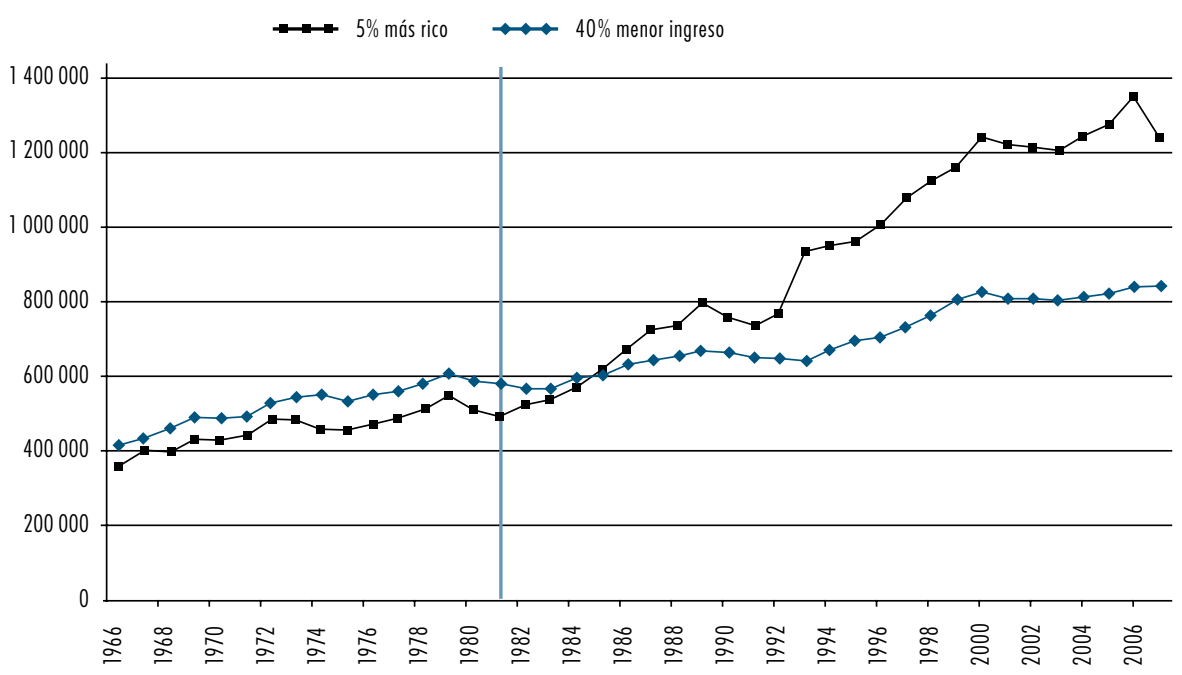

Fuente: elaboración propia con datos del US Census Bureau. Ingresos promedio por familia y número de familias por quintil provienen respectivamente de

http://www.census.gov/hhes/www/income/histinc/f03AR.html, y http://www.census.gov/hhes/www. income/histinc/f01AR.html. Cálculos propios del ingreso total a partir de los datos de dichas tablas.

\section{Conclusiones}

Esta rápida revisión de las estadísticas de los Estados Unidos de América nos muestra que durante los últimos 35 años ha habido un reducido crecimiento de los ingresos anuales reales del $40 \%$ de las familias norteamericanas pertenecientes a los dos primeros quintiles de población. En cambio, los grupos de mayor ingreso, en especial el 
de las familias blancas, cada vez han concentrado más riqueza en sus manos. Dicha concentración del ingreso en el $20 \%$ más rico de la población estadounidense ha limitado la capacidad de compra del mercado y ha obligado a financiar una gran parte del consumo con créditos que han superado la capacidad de pago de los prestatarios (James K. Galbraith).

La salud del mercado parte de una mejor distribución del ingreso y, por lo tanto, la concentración del ingreso atenta contra el mercado al impedir que la mayor parte de la población comparta el crecimiento del ingreso nacional. "La riqueza es central para la seguridad económica de los hogares" (Caner y Wolf, 2004: 1), ya que permite, entre muchas otras cosas, capacidad de ahorro y capacidad de crédito, y este último elemento ha mostrado su importancia en la actual crisis económica.

En este escrito he mostrado la existencia de un punto de quiebre (break point) en el año 1981 en las series de la evolución del ingreso medio por familia y de la concentración del ingreso en manos del 5\% y 20\% más rico de los EEUU. Este punto de quiebre coincide con la aplicación de las medidas neoliberales de reducción del Estado de bienestar y disminución de impuestos a los niveles mayores de ingresos. Al separar el antes y el después y ver sus tendencias, queda claro que hubo un cambio en la economía que impidió que la gran mayoría de la sociedad norteamericana compartiera el crecimiento económico. Lo anterior resulta más dramático si tomamos en cuenta que dicho punto de quiebre no aparece en la tendencia del crecimiento del PIB.

Hace dos años, en el otoño de 2007, escribí en el borrador de esta investigación: "Si tomamos en cuenta que en las naciones más pobres y más pobladas, ese mismo $40 \%$ de la población, no sólo no mantuvo sus ingresos sino que la pobreza los absorbió aún más, no podemos pensar en un panorama más preocupante para los próximos años en los que la reducción de la capacidad de compra de los mercados globales pueda generar una depresión generalizada del sistema, depresión que podría iniciarse con una crisis financiera".

Lo sucedido en el ínterin ha confirmado ampliamente aquel vaticinio. 


\section{Bibliografía}

Atkinson, A.B. y Thomas Piketty (eds.), Top Incomes. A Global Perspective, Oxford University Press, (en prensa)

Caner, Asena y Edward Nathan Wolf, "Asset poverty in the United States, 1984-1999: evidence from the Panel Study of Income Dynamics", Review of Income and Wealth, Blackwell Publishing, vol. 50 (4), diciembre de 2004.

Crown, William H. H. y Leonard F. Wheat, State Per-Capita Income Change since 1950: Sharecropping's Collapse and Other Causes of Convergence, Westport, Connecticut, Greenwood Press, 1995.

DeNavas-Walt, Carmen, Bernadette D. Proctor y Jessica C. Smith, "Income, poverty, and health insurance coverage in the United States: 2007”, Current Population Reports, Washington, DC, US Census Bureau, 2008 [http://www.census.gov/ prod/2008pubs/p60-235.pdf].

Dovring, Folke, Inequality: The Political Economy of Income Distribution, Nueva York, Praeger Publishers, 1991.

Encinas Ferrer, Carlos, "Distribución del ingreso y pobreza en los EEUU", Revista contexturas, León, Guanajuato, Universidad Iberoamericana León, 2000, pp. 13-18.

Encinas Ferrer, Carlos, Gerardo Reyes Guzmán y Luis Ignacio Román Morales, "Un planeta y cuatro o cinco mundos: la polarización económica y su impacto en el empleo", en Los rostros de la pobreza: El debate, tomo V, Guadalajara, ITESO, 2008, pp. 54-124.

Galbraith, James K., “A 'people first' strategy: Credit cannot flow when there are no creditworthy borrowers or profitable projects", The Levy Economics Institute of Bard College Strategic Analysis, abril de 2009 [http://www.levy.org/pubs/ sa_apr_09.pdf].

Gardels, Nathan, "Entrevista a Joseph Stiglitz", Reporte índigo, núm. 23, México, Indigo Brainmedia, 2007 [http://www.reporteindigo.com/pdf/23/Reporteindigo.pdf].

Kolko, Gabriel, Wealth and Power in America: An Analysis of Social Class and Income Distribution, Nueva York, Frederick A. Praeger, 1962.
Maxwell, Nan L., Income Inequality in the United States, 1947-1985, Nueva York, Greenwood Press, 1990.

Ryscavage, Paul, Income Inequality in America: An Analysis of Trends, Nueva York, M. E. Sharpe Armonk, 1999.

Saez, Emmanuel, "Striking it richer: The evolution of top incomes in the United States (update using 2006 preliminary estimates)", 15 de marzo de 2008 [http://elsa. berkeley.edu/ saez/saez-UStopincomes2006prel.pdf].

Stiglitz, Joseph, entrevista radiofónica realizada por Amy Goodman y Juan González, 5 de octubre de 2008 [http://www.sinpermiso.info/textos/index.php?id=2095].

The Levy Economics Institute of Bard College [http://www.levy.org/].

US Census Bureau: Home Page [http://www. census.gov].

US Census Bureau, Historical Income Tables-Families [http://www.census.gov/ hhes/www/ income/histinc/f01AR.html] y [http://www.census.gov/hhes/www/income/histinc/f03AR. html].

US Census Bureau, Poverty [http://www. census.gov/hhes/www/poverty/threshld/ thresh07. html].

US Census Bureau, Tablas F-3 all races y por raza [http://www.census.gov/hhes/www/ income/histinc/f03AR.html].

US Department of Labor, Bureau of Labor Statistics, Office of Productivity and Technology, "Comparative real gross domestic product per capita and per employed person: 16 countries 1960-2007”, Washington, D.C., Bureau of Labor Statistics, 7 de julio de 2008 [http:// www. bls.gov/fls/home.htm].

Zacharias, Ajit, Edward N. Wolff y Thomas Masterson, "New estimates of economic inequality in America, 1959-2004", Levy Institute Measure of Economic Well-Being, abril de 2009 [http://www.levy.org/ pubs/lmw_apr_09.pdf]. 